\title{
SURGERY
}

\section{Retroperitoneoscopic partial nephrectomy: practice is key}

A new paper in European Urology describes the outcomes of robot-assisted retroperitoneoscopic partial nephrectomy (RARPN) procedures performed by Jim Hu, Alon Weizer and James Porter at three clinics in the USA. These data represent the largest multicentre experience of RARPN to date, and provide insight into intersurgeon variability in outcomes.

Open surgery is the most-common approach to partial nephrectomy, despite the potential benefits of laparoscopy. However, transperitoneal laparoscopic partial nephrectomy is also less than ideal, requiring mobilization of the bowel and, for posterior renal tumours, full mobilization and flipping of the kidney. "Additionally, some institutions preferentially refer patients with posterior renal masses or those who have undergone prior abdominal surgery to radiofrequency ablation or cryotherapy (thermal ablation), despite the potential downsides to these percutaneous approaches," explains $\mathrm{Hu}$, "specifically, the lack of final pathology, and the increased use of additional post-treatment imaging for surveillance purposes, which increases radiation exposure and costs." The retroperitoneal approach might largely avoid these drawbacks, but has received comparatively less attention, possibly owing to limitations related to smaller working spaces and a lack of anatomical landmarks. "We demonstrate that this approach is feasible for posterior tumours or patients with prior abdominal surgery," states $\mathrm{Hu}$.

\section{$\mathbf{4}$ These data represent the largest multicentre experience of RARPN to date... 77}

After 227 consecutive RARPNs, 29 in patients who had undergone previous abdominal surgery, the frequencies of positive surgical margins and recurrence at a median of 2.7 years after surgery were $3.5 \%$ and $0.9 \%$, respectively; these rates are comparable with those published previously for alternative approaches to partial nephrectomy. Median operating time, warm ischaemia time (WIT) and estimated blood loss were $165 \mathrm{~min}, 19 \mathrm{~min}$, and $75 \mathrm{ml}$, respectively.

Overall, complications occurred in $12.3 \%$ of the patients, with a rate of intraoperative complication of $1.3 \%$. Importantly, "complications varied by more than threefold among surgeons and WIT varied by almost $5 \mathrm{~min}$, after adjusting for observed differences in patient and tumour characteristics," says Hu. These findings might, in part, reflect differences in the techniques used; however, statistical analyses revealed the a higher volume of surgical procedures was associated with decreases in the complication rates and WIT, suggesting that technical experience is an important determinant of RARPN outcomes.

David Killock

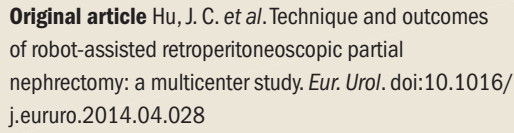

Original article $\mathrm{Hu}, \mathrm{J}$. C. et al. Technique and outcomes of robot-assisted retroperitoneoscopic partial nephrectomy: a multicenter study. Eur. Urol. doi:10.1016/ j.eururo.2014.04.028 